\title{
Enhancement of efficiency for broadband unidirectional light scattering by core-shell nanoantennas
}

\author{
Weimin Wang, Xiaoyue Wang, Biqun Huang, Xufeng Jing ${ }^{*}$, Chenxia Li ${ }^{*}$ \\ Institute of Optoelectronic Technology, China Jiliang University, Hangzhou 310018, China \\ ${ }^{*}$ Corresponding authors: jingxufeng_1984@163.com (Xufeng Jing), lichenxiacjlu@163.com (Chenxia Li)
}

\begin{abstract}
A core-shell nanocube antenna is proposed to numerically simulate broadband unidirectional scattering. Comparing the scattering of a dielectric nanocube and core-shell nanocube, it is found that the core-shell nanocube has a higher forward/backward ratio and better unidirectional scattering efficiency. In order to further enhance forward scattering efficiency and reduce the side-lobe of scattering, the particle chain of core-shell nanocubes is proposed by arranging multiple nanoparticles. It is possible to achieve better unidirectional scattering over a wide spectral range. In addition, we also numerically studied the effect of the gap between the particles and the radius of the metal core on unidirectional scattering. It is demonstrated that the optimal gap should be selected to achieve ideal unidirectional forward scattering. The unique optical properties of core-shell nanocubes have important practical applications particularly in the fields of nanoantennas, photovoltaic devices, and nanolasers that require reflection suppression.
\end{abstract}

Keywords: nanoparticles, core-shell nanocube, particle chains, broadband unidirectional scattering.

\section{Introduction}

On the account of the development of nanotechnology, the ability to manipulate light at the nanoscale is of great importance for future optical chip integration. Recently, many novel light scattering phenomena for nanoparticles at nanoscale, such as cloaking [1] , superscattering $[\underline{2}, \underline{3}]$ and directional light scattering $[\underline{4}, \underline{5}]$, as well as applications in biomedical treatment and imaging [ $\underline{6}-\underline{9}]$, can be realized $[\underline{1}, \underline{10}, \underline{11}]$. Furthermore, nanoparticles have been widely used in biomedical markers [12-15], and their influence on biology and medical research is very important. The research on unidirectional scattering applied to nanoantennas catches people's attention at present. Although there are many researches on unidirectional scattering at present, how to achieve high-efficiency, broadband, and super-orientation are the current research directions. Plasma nanoparticles made of precious metals have been shown to be potential cornerstones of nanoantennas $[\underline{16}, \underline{17}]$, but the diffraction limit and significant energy losses are unavoidable, especially at visible wavelengths. In addition, it has also been found that 
the dimer of silicon nanostructures can also achieve directional scattering effect [18]. But because of its limited tuning ability, it cannot achieve very good results and generally only works in the optical band.

To suppress the unwanted backward scattering and enhance the directional forward scattering, an extra reflector coupled item [19-22], Fabry-Pérot resonator-like structures $[\underline{23}, \underline{24}]$ or complicated structure engineering [ $\underline{25}-\underline{27}]$ are usually employed. It is theoretically predicted that magnetic particles can exhibit unidirectional scattering without involving complex structures or additional reflectors. A core-shell nanoparticle can support both electrical and magnetic resonances, and when the resonance design is reasonable, it is expected to achieve a very good broadband directional scattering effect. Also, it can be applied in the fields of nanoantennas, photovoltaic devices, and nano-sized lasers that require backscatter suppression.

Here, we propose a cubic dielectric nanoparticle containing a single metal particle for broadband unidirectional scattering. Due to a larger optical coefficient, larger packing density, and relative ease of fabrication compared with spherical nanoparticles, it can be a promising candidate for future low-loss, ultra-compact nanophotonic applications. This core (metal)-shell (dielectric) nanocube nanoantenna is demonstrated to achieve high-efficiency unidirectional light scattering. We compared the scattering of dielectric silicon and core-shell nanocube to illustrate the effect of metal core on scattering and the effect of changing the metal core size on directional scattering. Finally, we used the core-shell nanocube particle chains to suppress backscattering and enhance forward scattering, which provides a good solution and idea for nanoantenna designers.

\section{Simulation results and discussions}

\subsection{Numerical analysis and comparison of dielectric nanocube and core-shell nanocube}

We numerically simulated a core (metal)-shell (dielectric) nanocube particle in the air using the finite-difference time-domain (FDTD) method. The numerical results of the forward and backward scattering spectra, their ratio (F/B ratio) and the modal field distribution inside the particles are based on the Maxwell equations. A wide-band $(\lambda=400-1000 \mathrm{~nm})$ plane wave is launched with normal incidence, and the field monitor is located in the particle and around the particle to monitor the local field strength. A 2.5-nm refined mesh was applied around the particles when monitoring the near-field intensity, and the perfectly matched layer (PML) was used for the boundary conditions. Moreover, the far-field scattering was simulated by the finite integral method.

Figure 1 shows the numerical results of the forward and backward scattering spectra and F/B ratio of a dielectric nanocube and core-shell nanocube. Note that we integrated the scattered field separately in the forward and backward directions to calculate its forward and backward scattering cross-sections. We used silicon as the dielectric shell with the refractive index $n=3.4$, and its length and width are $700 \mathrm{~nm}$, and its height is $200 \mathrm{~nm}$. The material of the metal core is silver, and its diameter is $d=150 \mathrm{~nm}$. For the permittivity of silver, the experimental data from [28] was used. In terms of 

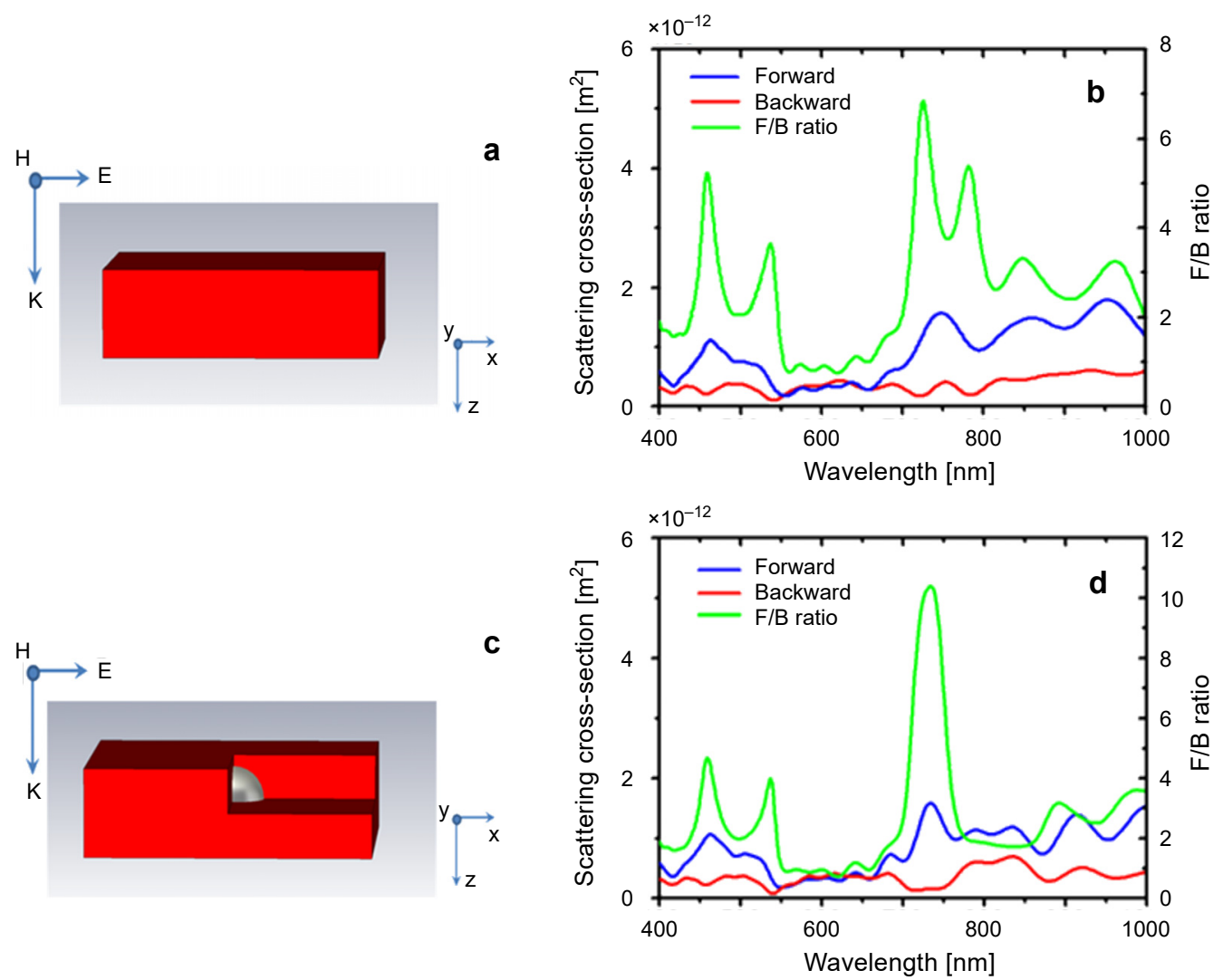

Fig. 1. Scattering of incident plane waves by dielectric silicon cube (a). Spectra of the forward (F) and backward (B) scattering cross-section of dielectric silicon and F/B ratio (b). Scattering of incident plane waves by a core-shell nanocube (c). Spectra of the forward and backward scattering cross-sections of core -shell nanocube and the ratio of $\mathrm{F} / \mathrm{B}(\mathbf{d})$.

theoretical analysis, when the structure is illuminated by a plane wave, the scattered light is collected to the far field. In Fig. 1b, we firstly simulated a silicon nanocube, and the plane wave is incident perpendicularly from the top. It can be found that the F/B ratio of silicon nanocube is less than 8 , and the forward and backward scattering cross-sections are relatively small. The scattering efficiency is relatively poor, and the requirement of directional scattering cannot be achieved. In Fig. 1d, the F/B ratio of the core -shell nanocube is approximate 11, demonstrating forward scattering by unidirectional scattering and high scattering efficiency compared to silicon nanocube. This $\mathrm{F} / \mathrm{B}$ is greatly higher than that of two different dielectric nanospheres [18]. Therefore, it can be considered that when $\mathrm{F} / \mathrm{B}$ is higher than 8 , it is a wide-band directional scattering device in the range of $710-750 \mathrm{~nm}$, and the directional scattering of the device, especially at $740 \mathrm{~nm}$, achieves optimal performance.

In the F/B ratio spectrum of the silicon nanocube in Fig. 1b, a maximum occurs at $\lambda=725 \mathrm{~nm}$. We can find out that the silicon nanocube has both electric resonance and 

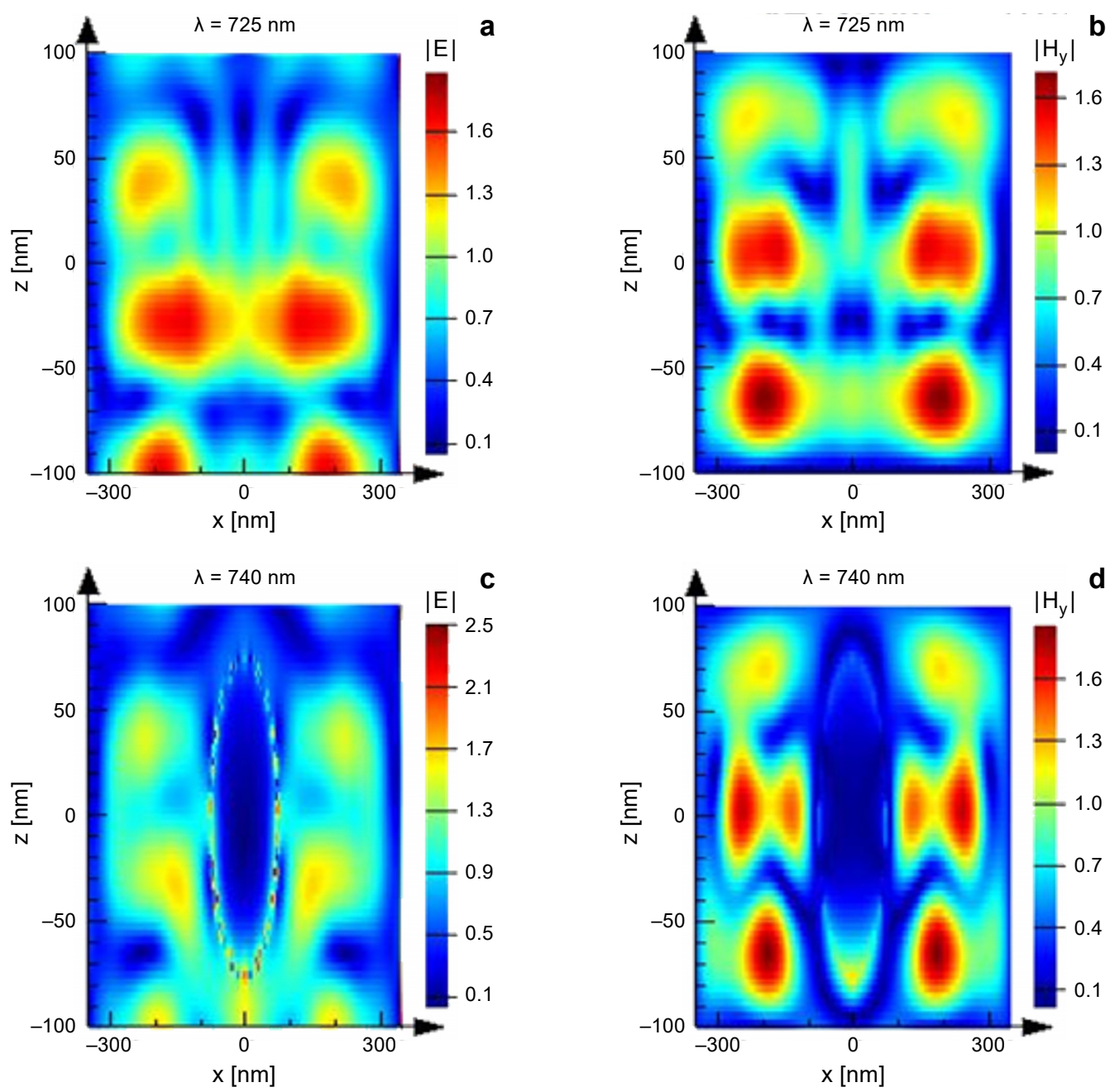

Fig. 2. The electric field intensity $|E|$ distribution (a) and the magnetic field intensity $\left|H_{y}\right|$ distribution (b) of silicon nanocube in the $x-z$ plane at $\lambda=725 \mathrm{~nm}$. The electric field intensity $|E|$ distribution (c) and the magnetic field intensity $\left|H_{y}\right|$ distribution (d) of core-shell nanocube in the $x$-z plane at $\lambda=740 \mathrm{~nm}$. The units for the electric and magnetic fields are $\mathrm{V} / \mathrm{m}$ and $\mathrm{A} / \mathrm{m}$, respectively.

magnetic resonance at $\lambda=725 \mathrm{~nm}$ by the electromagnetic field diagram in Figs. 2a and $2 \mathbf{b}$. At the same time, at $\lambda=725 \mathrm{~nm}$, the electrical resonance is equal to the magnetic resonance and satisfies the first Kerker condition, so directional scattering can be generated. However, since the Kerker condition is far from the formant, the scattering efficiency in the forward direction at these wavelengths is very low. The core-shell nanocube shows a clear new peak in the F/B ratio spectrum around $\lambda=740 \mathrm{~nm}$. The forward scattering exhibits a peak, and backscattering is suppressed. Core-shell nanoparticles 
can be effectively viewed as a pair of orthogonal electric dipoles and a combination of magnetic dipoles. We note here that the backscattering of the core-shell nanoparticles is almost completely suppressed at $\lambda=740 \mathrm{~nm}$ due to the fact that the wavelength of the unidirectional scattering of the core-shell nanoparticles is close to the wavelength of the magnetic dipole resonance and the electric dipole resonance.

Figures $3 \mathbf{b}$ and $3 \mathbf{d}$ show the cross-section of the radiation pattern of the scattered field at the maximum $\mathrm{F} / \mathrm{B}$ ratio wavelength in the $x-z$ plane. The scattering intensity in the $180^{\circ}$ direction corresponds to direct forward scattering. Figure $3 \mathbf{b}$ clearly shows
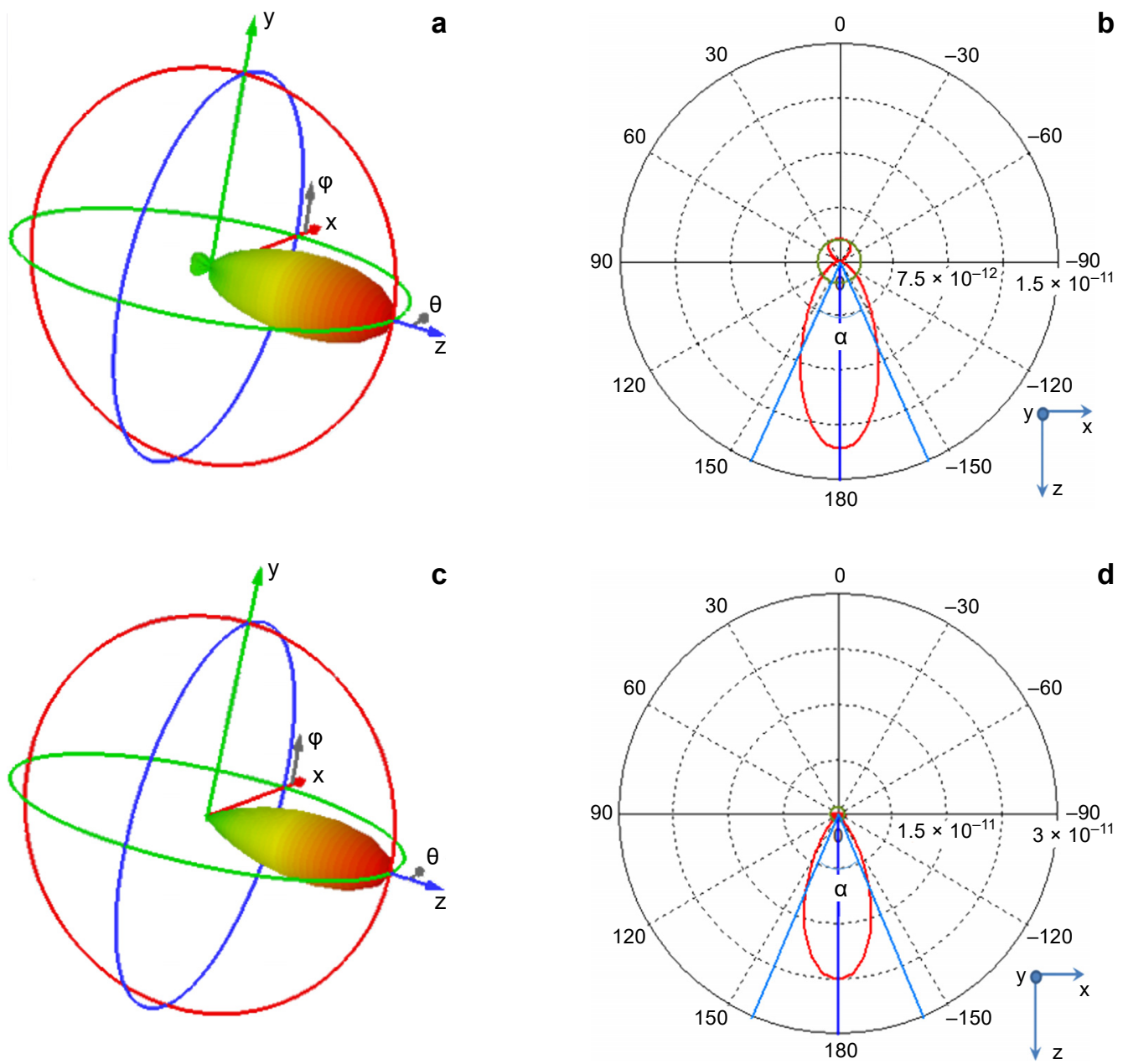

Fig. 3. Three-dimensional far-field scattering of silicon nanocube at wavelength $\lambda=725 \mathrm{~nm}$ (a) and silicon nanocube scattering mode in the far field (b). 3D far-field scattering of the core-shell nanocube at wavelength $\lambda=740 \mathrm{~nm}$ (c) and core-shell nanocube scattering mode in the far field (d). The main lobe beam width $\alpha$ is defined and shown in (b) and (d). 


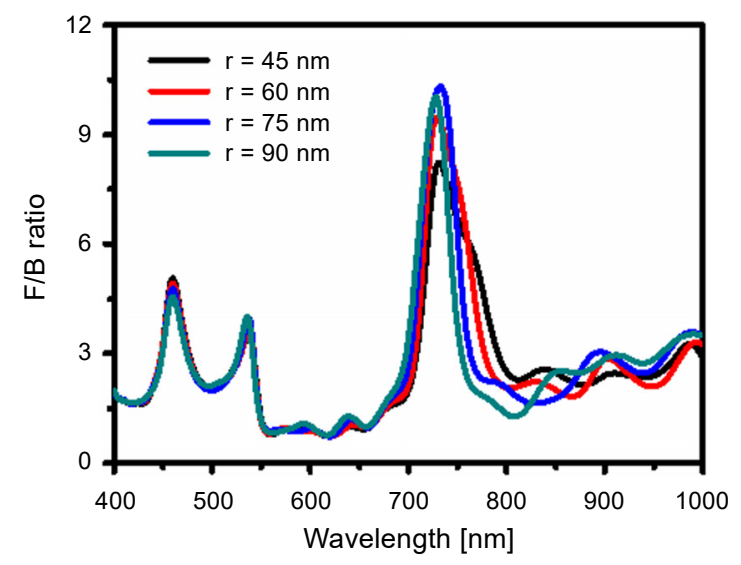

a
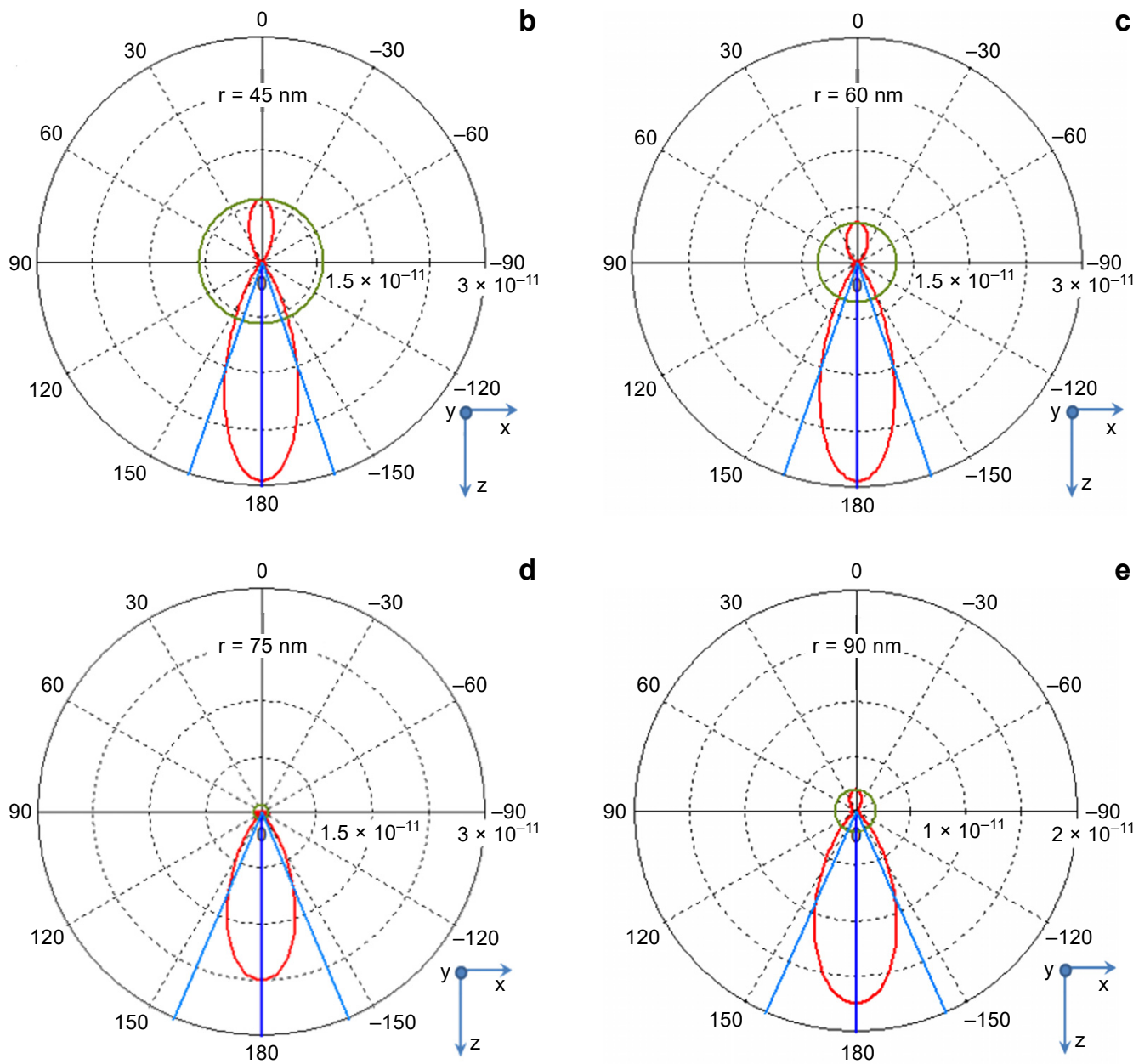

Fig. 4. F/B ratios of core-shell nanocube for different metal core radii (a). Scattering patterns for metal core radius $r=45 \mathrm{~nm}(\mathbf{b}), r=60 \mathrm{~nm}(\mathbf{c}), r=75 \mathrm{~nm}(\mathbf{d})$, and $r=90 \mathrm{~nm}(\mathbf{e})$, when $\lambda=740 \mathrm{~nm}$. 
that backscattering of silicon nanocube is not negligible because of its high intensity and the presence of energy in both forward and backward scattering. Energy leakage of backscattering will be a great impact on practical applications. However, it can be seen that the backscattering intensity of the core-shell nanocube is small, and the intensity of forward scattering increases. This strength enhancement clearly shows the advantages of the core-shell type nanocube, and it can make full use of scattering resonance for unidirectional forward scattering. In fact, we expect efficient unidirectional forward scattering and prevent the generation of side lobes. Next we will use core-shell nanoparticle chains to achieve a smaller main lobe beam width $\alpha$.

In addition, we also discuss the influence of metal core radius on the scattering of the core-shell nanocube. We notice that metal nuclei of not all sizes contribute to the forward scattering of particles. In Fig. 4, the effect of metal core radius on nuclear-nanoparticle scattering is demonstrated.

Comparing the $\mathrm{F} / \mathrm{B}$ ratio of different $\mathrm{Ag}$ radii in Fig. $4 \mathbf{a}$, we find that the $\mathrm{F} / \mathrm{B}$ value is obviously larger than other cases when $r=75 \mathrm{~nm}$ near $\lambda=740 \mathrm{~nm}$ even though the F/B peaks of different Ag radii are different. From Figs. $4 \mathbf{b}-4 \mathbf{e}$ it can be found that when the radius of Ag core is $r=75 \mathrm{~nm}$ at $\lambda=740 \mathrm{~nm}$, it has the best forward-scattering and suppressing backward-scattering. On the contrary, when the particle radius is too small or too large, the backscattering will increase, which leads to the deterioration of the scattering orientation of the device.

\subsection{Scattering of core-shell nanocube chains}

The theoretical model for the coupling between particles can be adopted from a generalized model describing the interaction of $N$-particle linear chains. The effective electrical and magnetic dipole polarizability can be expressed as [29]

$$
\begin{aligned}
& \alpha_{1}^{\mathrm{e}}=\frac{3 i}{2 k^{3}} a_{1} \\
& \alpha_{1}^{\mathrm{m}}=\frac{3 i}{2 k^{3}} b_{1}
\end{aligned}
$$

where $a_{1}$ and $b_{1}$ are the Mie scattering coefficients, corresponding to the electrical dipole moment and the magnetic dipole moments, respectively, and $k$ is the angular wave number in the background material.

From the theoretical study of the scattering of the chains, each core-shell type nanoparticle can be treated as a combination of orthogonal electric dipole (ED) and magnetic dipole (MD) resonances, which has the polarizability described by Eq. (1). Based on the coupled dipole approximation, the electrical $d_{i}$ and magnetic $m_{i}$ dipole moments of the $i$-th nanocube in the $N$-nanotube chain can be expressed as [ $\underline{30}, \underline{31}]$

$$
d_{i}=\alpha_{1}^{\mathrm{e}} E_{i}^{0}+\alpha_{1}^{\mathrm{e}} \sum_{j=1: N}^{j \neq i}\left(E_{i}^{d_{j}}+E_{i}^{m_{j}}\right)
$$



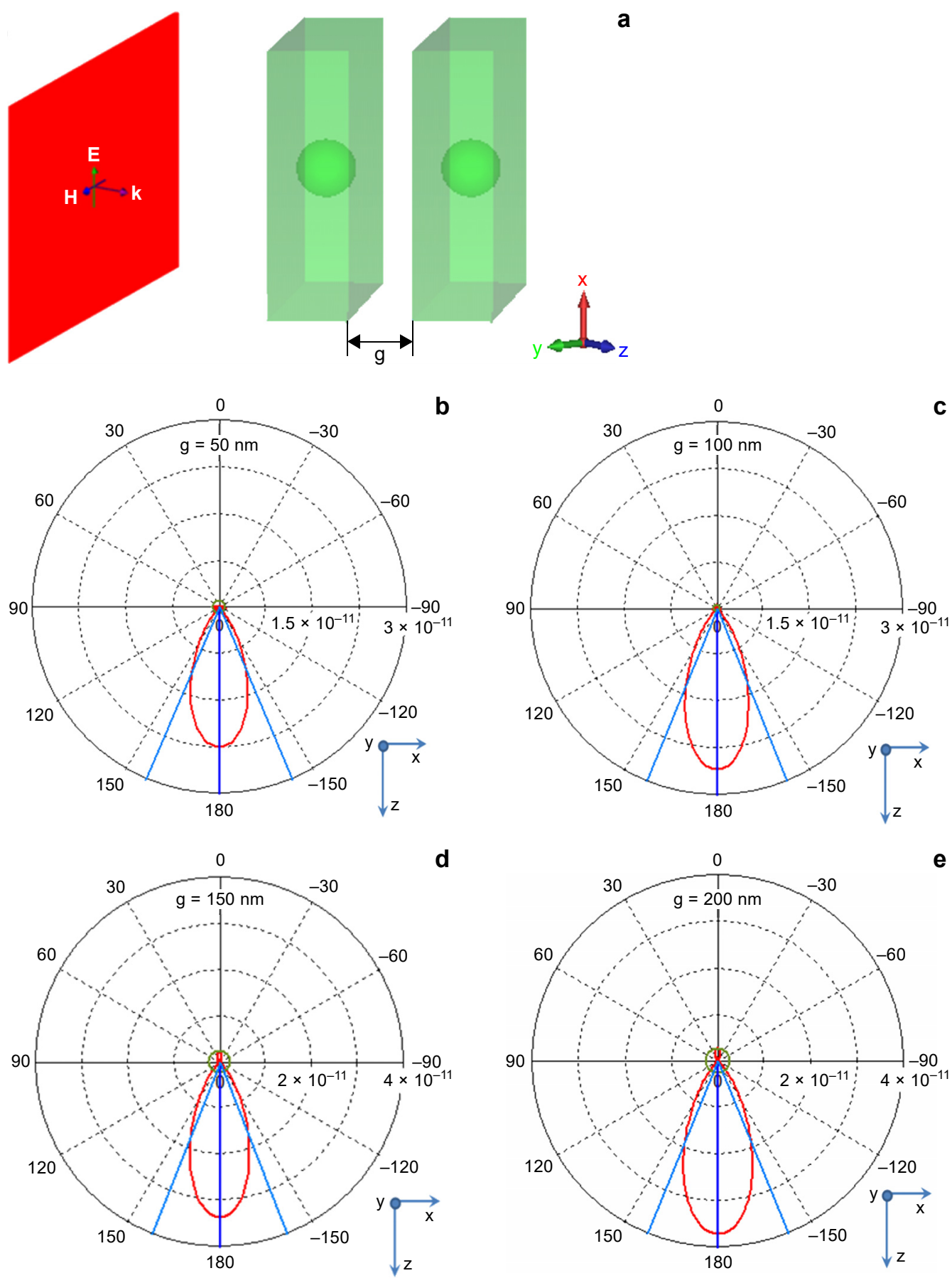

Fig. 5. Comparison of a core-shell nanocube dimer with different gap. Light scattering diagram of dimer particles (a). Directional scattering of dimer particles at different gap $g=50 \mathrm{~nm}(\mathbf{b}), g=100 \mathrm{~nm}(\mathbf{c})$, $g=150 \mathrm{~nm}(\mathbf{d})$, and $g=200 \mathrm{~nm}(\mathbf{e})$, at $\lambda=740 \mathrm{~nm}$. 


$$
m_{i}=\alpha_{1}^{\mathrm{m}} H_{i}^{0}+\alpha_{1}^{\mathrm{m}} \sum_{j=1: N}^{j \neq i}\left(H_{i}^{d_{j}}+H_{i}^{m_{j}}\right)
$$

where $E_{i}^{0}$ and $H_{i}^{0}$ are the electric and magnetic fields of the incident wave at position $r_{i}$, respectively; $E_{i}^{d_{j}}$ and $H_{i}^{d_{j}}$ are the electric and magnetic fields of $d_{i}$ radiation at $r_{i}$, respectively; $E_{i}^{m_{j}}$ and $H_{i}^{m_{j}}$ is the radiation field of $m_{j}$ at $r_{i}$. By solving Eqs. (2) and (3), we can obtain the electric and magnetic moments of each particle, and then we can find that the scattering pattern is a superposition of the radiation of all $2 N$ interaction dipole moments.

Here, we first consider the simple case of the core-shell nanocube dimer and then thoroughly investigate the effect of particle gaps on scattering. In general, nanodimers have better directional scattering than single particles. Figure 5 shows the effect of gap between particles on scattering by comparing four scattering patterns at different gaps $g=50 \mathrm{~nm}, 100 \mathrm{~nm}, 150 \mathrm{~nm}$, and $200 \mathrm{~nm}$. From Fig. 5b, we can see that at $g=50 \mathrm{~nm}$, the interaction of the two particles causes the side-scattering to appear in the forward scattering due to the small gap; when $g>150 \mathrm{~nm}$, the scattering pattern shows a more obvious backscattering. However, it has the best directional scattering and suppresses backscattering at $g=100 \mathrm{~nm}$. We can find that a smaller gap leads to poorer directionality and side lobes, and a larger gap increases the forward scattering intensity but also increases the intensity of backscattering. So the gap $g=100 \mathrm{~nm}$ should be chosen as the scattering case when the number of analyzed particles is more than two as $N>2$.

In Figs. 6 and 7, we study the unidirectional scattering patterns of different numbers of particles in the chain. It can be clearly seen from Fig. 7 that by increasing the number of particles, the main lobe beam width will be reduced, the forward scattering will be enhanced and the directionality will be better. From Fig. 7, it can be seen that the main lobe beam width $\alpha$ is already less than $30^{\circ}$ when the number of particles is $N=4$, and it has the largest forward scattering intensity, which means that by increasing the number of particles, it can play a role in concentrating, but the backscattering also increases slightly. We do not continue to increase the number of particles $N$. In practical appli-
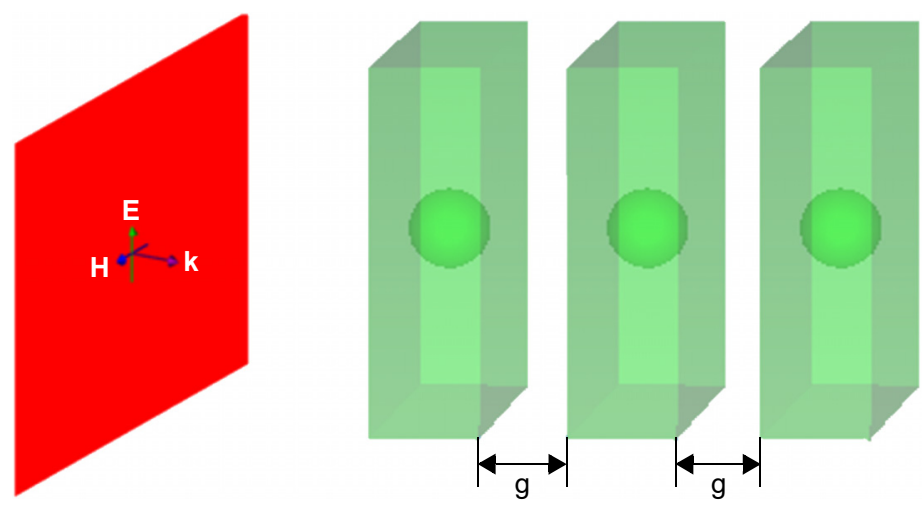

a

Fig. 6. Light scattering diagram of a core-shell nanoparticle chain with gap $g$. 

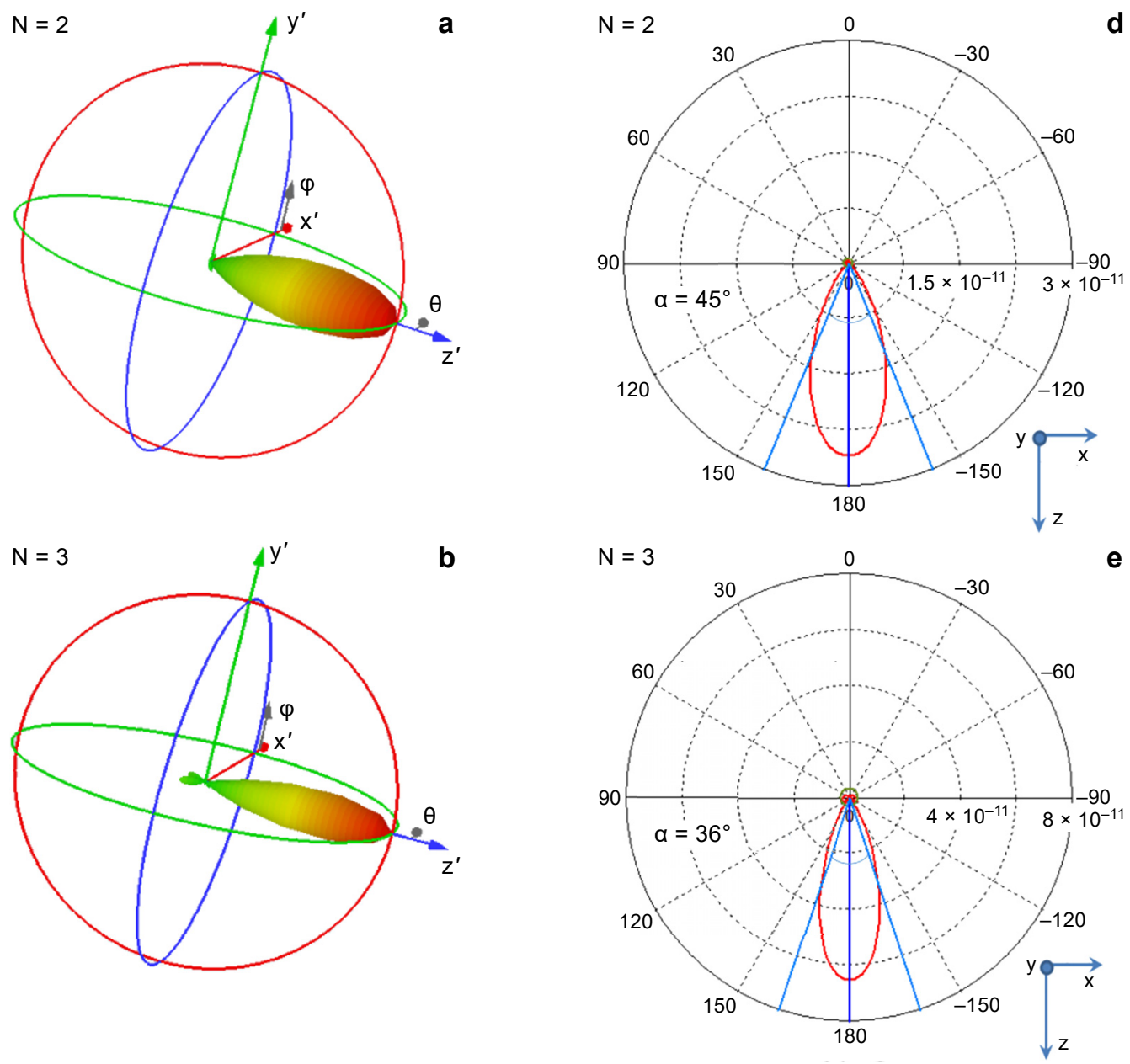

b
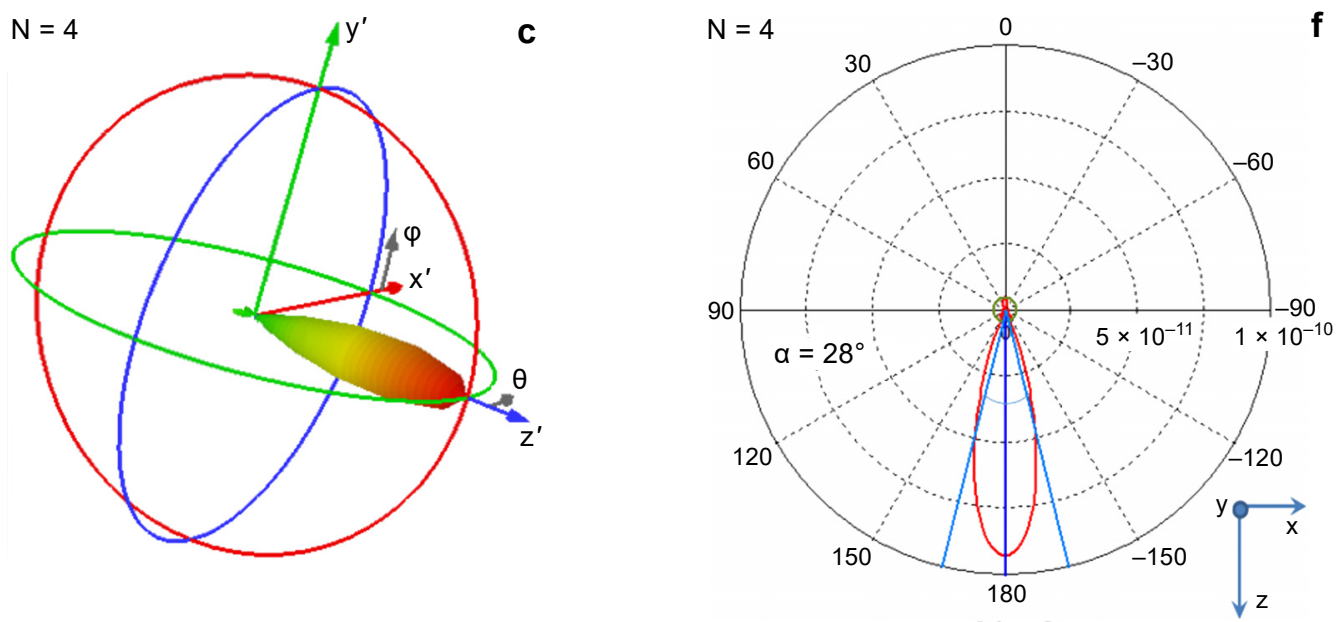

Fig. 7. The 3D far-field scattering $(\mathbf{a}-\mathbf{c})$ and scattering patterns $(\mathbf{d}-\mathbf{f})$ at fixed distance $g=100 \mathrm{~nm}$. 
cations, the fewer the number of particles, the simpler the structure, and too many particles is impractical, so we choose the maximum number of particles as $N=4$. It should be noted here that the mechanism of enhancing the directivity of the nanoparticle array is basically the same as the mechanism of the Yagi-Uda antennas, in which the interference plays a major role.

\section{Conclusion}

In conclusion, we systematically studied the scattering properties of (metal) core-(dielectric) shell nanoparticles and showed that they have good directional scattering. Core-shell nanocube has better directional scattering patterns than silicon nanocube, and it has higher F/B value and good directional scattering around $40 \mathrm{~nm}$ bandwidth. We also numerically calculated the effect of particle gap on scattering, and it was revealed that the best forward scattering effect can be obtained when $g=100 \mathrm{~nm}$. We have further shown that the directionality of a single core-shell nanoparticle scattering can be enhanced when the nanoparticles are arranged in a one-dimensional chain. As the number of particle chains increases, the beam width of the main lobe decreases. Compared with the scattering of a single core-shell nanoparticle, the directional scattering can be significantly improved by increasing the number of particle chains. At the same time, we analyzed the effect of different metal core sizes on the scattering pattern with other parameters unchanged. The broadband high efficient unidirectional scattering of core -shell nanoparticles can be applied in nanoantenna, nanoscale lasers, photovoltaics and even biomedical applications, and so on [32-40].

Acknowledgments - The authors acknowledge the support from Natural Science Foundation of Zhejiang Province (LY20F050007), National Natural Science Foundation of China (NSFC) (No. 61405182, and No. 61377108).

\section{References}

[1] Alu A., Engheta N., Cloaking a sensor, Physical Review Letters 102(23), 2009, article ID 233901, DOI: 10.1103/PhysRevLett.102.233901.

[2] Ruan Z., FAn S., Superscattering of light from subwavelength nanostructures, Physical Review Letters 105(1), 2010, article ID 013901, DOI: 10.1103/PhysRevLett.105.013901.

[3] Chenchen Zhao, Ji Chen, Hanmeng Li, Tao Li, Shining Zhu, Mode division multiplexed holography by out-of-plane scattering of plasmon/guided modes, Chinese Optics Letters 16(7), 2018, article ID 070901.

[4] Xinguie Ni, Emani N.K., Kildishev A.V., Boltasseva A., Shalaev V.M., Broadband light bending with plasmonic nanoantennas, Science 335(6067), 2012, p. 427, DOI: 10.1126/science.1214686.

[5] Chen W., Zhang J., Peng B., Özdemir Ş.K., Fan X., Yang L., Parity-time-symmetric whispering-gallery mode nanoparticle sensor, Photonics Research 6(5), 2018, pp. A23-A30, DOI: 10.1364/PRJ.6.000A23.

[6] Sikdar D., Rukhlenko I.D., Cheng W., Premaratne M., Effect of number density on optimal design of gold nanoshells for plasmonic photothermal therapy, Biomedical Optics Express 4(1), 2013, pp. 15-31, DOI: 10.1364/BOE.4.000015.

[7] Sikdar D., Rukhlenko I.D., Cheng W., Premaratne M., Tunable broadband optical responses of substrate-supported metal/dielectric/metal nanospheres, Plasmonics 9(3), 2014, pp. 659-672, DOI: 10.1007/s11468-014-9681-8. 
[8] Chou R.Y., Lu G., Shen H., He Y., Cheng Y., Perriat P., Martini M., Tillement O., Gong Q., A hybrid nanoantenna for highly enhanced directional spontaneous emission, Journal of Applied Physics 115(24), 2014, article ID 244310, DOI: 10.1063/1.4885422.

[9] Wei Liu, Jianfa Zhang, Bing Lei, Haotong Ma, Wenke Xie, Haojun Hu, Ultra-directional forward scattering by individual core-shell nanoparticles, Optics Express 22(13), 2014, pp. 16178-16187, DOI: $10.1364 / \mathrm{OE} .22 .016178$.

[10] Alu A., Engheta N., Tuning the scattering response of optical nanoantennas with nanocircuit loads, Nature Photonics 2, 2008, pp. 307-310, DOI: 10.1038/nphoton.2008.53.

[11] Daquan Yang, Fei Gao, Qi-Tao Cao, Chuan Wang, Yuefeng Ji, Yun-Feng Xiao, Single nanoparticle trapping based on on-chip nanoslotted nanobeam cavities, Photonics Research 6(2), 2018, pp. 99-108, DOI: 10.1364/PRJ.6.000099.

[12] Atwater H.A., Polman A., Plasmonics for improved photovoltaic devices, Nature Materials 9, 2010, pp. 205-213, DOI: $10.1038 /$ nmat2629.

[13] Sikdar D., Rukhlenko I.D., Cheng W., Premaratne M., Optimized gold nanoshell ensembles for biomedical applications, Nanoscale Research Letters 8, 2013, pp. 142-146, DOI: 10.1186/1556-276X $-8-142$.

[14] Xue Han, Viet Giang Truong, Prince Sunil Thomas, Síle Nic Chormaic, Sequential trapping of single nanoparticles using a gold plasmonic nanohole array, Photonics Research 6(10), 2018, pp. 981 -986, DOI: 10.1364/PRJ.6.000981.

[15] Alavirad M., Olivieri A., Roy L., Berini P., Fabrication of electrically contacted plasmonic Schottky nanoantennas on silicon, Chinese Optics Letters 16(5), 2018, article ID 050007.

[16] Maier S., Plasmonics: Fundamentals and Applications, Springer, New York, 2007.

[17] Gramotnev D.K., Bozhevolny S.I., Plasmonics beyond the diffraction limit, Nature Photonics 4, 2010, pp. 83-91, DOI: 10.1038/nphoton.2009.282.

[18] Shibanuma T., Albella P., Maier S.A., Unidirectional light scattering with high efficiency at optical frequencies based on low-loss dielectric nanoantennas, Nanoscale 8(29), 2016, pp. 14184-14192, DOI: $10.1039 / \mathrm{C} 6 \mathrm{NR} 04335 \mathrm{~F}$.

[19] Curto A.G., Volpe G., Taminiau T.H., Kreuzer M.P., Quidant R., van Hulst N.F., Unidirectional emission of a quantum dot coupled to a nanoantenna, Science 329(5994), 2010, pp. 930-933, DOI: 10.1126/science.1191922.

[20] Kosako T., Kadoya Y., Hofmann H.F., Directional control of light by a nano-optical Yagi-Uda antenna, Nature Photonics 4, 2010, pp. 312-315, DOI: 10.1038/nphoton.2010.34.

[21] Pakizeh T., Kall M., Unidirectional ultracompact optical nanoantennas, Nano Letters 9(6), 2009, pp. 2343-2349, DOI: 10.1021/n1900786u.

[22] Evlyukhin A.B., Bozhevolnyi S.I., Pors A., Nielsen M.G., Radko I.P., Willatzen M., Albrektsen O., Detuned electrical dipoles for plasmonic sensing, Nano Letters 10(11), 2010, pp. 4571-4577, DOI: $10.1021 / \mathrm{nl} 102572 \mathrm{q}$.

[23] Shegai T., Miljkovic V.D., Bao K., Xu H., Nordlander P., Johansson P., Kall M., Unidirectional broadband light emission from supported plasmonic nanowires, Nano Letters 11(2), 2011, pp. 706-711, DOI: $10.1021 / \mathrm{nl} 103834 \mathrm{y}$.

[24] Zhipeng Li, Feng Hao, Yingzhou Huang, Yurui Fang, Nordlander P., Hongxing Xu, Directional light emission from propagating surface plasmons of silver nanowires, Nano Letters 9(12), 2009, pp. 4383-4386, DOI: $10.1021 / \mathrm{n} 1902651 \mathrm{e}$.

[25] Aouani H., Mahboub O., Bonod N., Devaux E., Popov E., Rigneault H., Ebbesen T.W., Wenger J., Bright unidirectional fluorescence emission of molecules in a nanoaperture with plasmonic corrugations, Nano Letters 11(2), 2011, pp. 637-644, DOI: 10.1021/nl103738d.

[26] Mian Wang, Cheng Yin, Yougiao Ma, Jun Zhou, Hanhua Zhong, Xianfeng Chen, Polarization and structure dependent optical characteristics of the three-arm nanoantenna with C3v symmetry and broken symmetry, Chinese Optics Letters 16(5), 2018, article ID 052501. 
[27] Bin Zhuang, Chengfang Xu, Yi Geng, Guangzhi Zhao, Hui Chen, Zhengquan He, Zhaoxin Wu, LIYONG REN, Round-trip imaging through scattering media based on optical transmission matrix, Chinese Optics Letters 16(4), 2018, article ID 041102.

[28] Johnson P.B., Christy R.W., Optical constants of the noble metals, Physical Review B 6(12), 1972, pp. 4370-4379, DOI: 10.1103/PhysRevB.6.4370.

[29] Bohren C.F., Huffman D.R., Absorption and Scattering of Light by Small Particles, Wiley, New York, 1983.

[30] Mulholland G.W., Bohren C.F., Fuller K.A., Light-scattering by agglomerates: coupled electric and magnetic dipole method, Langmuir 10(8), 1994, pp. 2533-2546, DOI: 10.1021/la00020a009.

[31] Merchiers O., Moreno F., Gonzalez F., Saiz J.M., Light scattering by an ensemble of interacting dipolar particles with both electric and magnetic polarizabilities, Physical Review A 76(4), 2007, article ID 043834, DOI: 10.1103/PhysRevA.76.043834.

[32] Bo Fang, Xufeng Jing, Qiufeng, Ye, Jinhui Cai, Pengwei Zhou, Broadband electromagnetic dipole resonance by the coupling effect of multiple dielectric nanocylinders, Applied Sciences 8(1), 2018, article ID 60, DOI: 10.3390/app8010060.

[33] Xincui Gui, Xufeng Jing, Jianjun Liu, Pengwei Zhou, Zhi Hong, Broadband polarization-independent two-dimensionally isotropic ultrahigh index metamaterials, Infrared Physics and Technology 89, 2018, pp. 174-180, DOI: 10.1016/j.infrared.2018.01.013.

[34] Weimin Wang, Xufeng Jing, Jingyin Zhao, Yinyan Li, Ying Tian, Improvement of accuracy of simple methods for design and analysis of a blazed phase grating microstructure, Optica Applicata 47(2), 2017, pp. 183-198, DOI: 10.5277/oa170202.

[35] Rui Xia, Xufeng Jing, Huinui Zhu,Weimin Wang, Ying Tian, Zhi Hong, Broadband linear polarization conversion based on the coupling of bilayer metamaterials in the terahertz region, Optics Communications 383, 2017, pp. 310-315, DOI: 10.1016/j.optcom.2016.08.060.

[36] Jingyin Zhao, Xufeng Jing, Weimin Wang, Ying Tian, Dongshuo Zhu, Guohua Shi, Steady method to retrieve effective electromagnetic parameters of bianisotropic metamaterials at one incident direction in the terahertz region, Optics and Laser Technology 95, 2017, pp. 56-62, DOI: 10.1016/ j.optlastec.2017.04.001.

[37] XUfEng Jing, XINCUI GUI, RUI XIA, ZHI Hong, Ultrabroadband unnaturally high effective refractive index metamaterials in the terahertz region, IEEE Photonics Journal 9(1), 2017, article ID 59001077, DOI: 10.1109/JPHOT.2016.2647558.

[38] Xufeng Jing, Rui Xia, Weimin Wang, Ying Tian, Zhi Hong, Determination of the effective constitutive parameters of bianisotropic planar metamaterials in the terahertz region, Journal of the Optical Society of America A 33(5), 2016, pp. 954-961, DOI: 10.1364/JOSAA.33.000954.

[39] Rui Xia Xufeng Jing, Xincui Gui, Ying Tian, Zhi Hong, Broadband terahertz half-wave plate based on anisotropic polarization conversion metamaterials, Optical Materials Express 7(3), 2017, pp. 977 -988, DOI: 10.1364/OME.7.000977.

[40] Huinui Zhu, Xufeng Jing, Pengwei Zhou, Strong dipole and higher multi-pole Mie resonance modes with all-dielectric nanoring metasurfaces structure, Superlattices and Microstructures 113, 2018, pp. 592-599, DOI: 10.1016/j.spmi.2017.11.045.

Received September 10, 2018 in revised form December 12, 2018 